\title{
Giant spin-orbit interactions in argon photoionization
}

\author{
D. H. Jaecks, ${ }^{1}$ O. Yenen, ${ }^{1}$ K. W. McLaughlin, ${ }^{2}$ S. Canton, ${ }^{3}$ J. D. Bozek, ${ }^{4}$ and M. Downsbrough ${ }^{1}$ \\ ${ }^{1}$ Behlen Laboratory of Physics, University of Nebraska-Lincoln, Lincoln, Nebraska 68588-0111, USA \\ ${ }^{2}$ Department of Physics and Engineering, Loras College, Dubuque, Iowa 52004-0178, USA \\ ${ }^{3}$ Department of Physics, Western Michigan University, Kalamazoo, Michigan 49008, USA \\ ${ }^{4}$ Advanced Light Source, Ernest Orlando Berkeley National Laboratory, University of California, Berkeley, California 94720 , USA
}

(Received 19 February 2004; published 29 October 2004)

\begin{abstract}
We have measured the relative cross sections for quartet and doublet satellite states of $\mathrm{Ar}^{+}$formed in photoionization. Quartet states are dipole forbidden in the nonrelativistic limit and result from spin-orbit interactions in the continuum [H. W. Van der Hart and C. H. Greene, J. Phys. B 32, 4029 (1999)]. Because of this, the cross sections for their formation are generally thought to be smaller than the dipole-allowed doublet states. We find that the cross sections for the $3 p^{4}\left[{ }^{3} P\right] 4 p{ }^{4} D_{1 / 2}^{o}$ and $3 p^{4}\left[{ }^{3} P\right] 4 p{ }^{4} D_{3 / 2}^{o}$ quartet states over certain energy regions are 16 to 30 times larger than doublet states. This counterintuitive result remains to be theoretically explained.
\end{abstract}

DOI: 10.1103/PhysRevA.70.040703

PACS number(s): $32.80 . \mathrm{Fb}$

A clear signature of spin-orbit interactions in the photoionization of $\mathrm{Ar}$ is the formation of quartet states of $\mathrm{Ar}^{+}$. The proposed principal mechanism by which these states are formed is one where the dominant dipole transitions to the ${ }^{1} P_{1}^{o}$ component of the intermediate Ar state formed just after the absorption of the photon are mixed by spin-orbit coupling with ${ }^{3} S_{1}^{o},{ }^{3} P_{1}^{o}$, and ${ }^{3} D_{1}^{o}$ components [1]. Quintet states are also possible but have not been considered in any calculation in the energy region considered in the present experiment. The triplet components of the intermediate Ar state make spin-conserving autoionizing transitions to quartet states of $\mathrm{Ar}^{+}$plus a free electron. The singlet component decays to the doublet states of $\mathrm{Ar}^{+}$plus a free electron. Previous measurements of the formation of quartet states suggest that the resonant states of $\mathrm{Ar}^{* *}$ are indeed dominated by the ${ }^{1} P_{1}^{o}$ state $[2,3]$. In this Rapid Communication we report on measurements where quartet cross sections are larger than the doublet cross sections, an unexpected result within the context of the present theoretical development [1].

The exceptions that we have found were in the formation of $3 p^{4}\left[{ }^{3} P\right] 4 p{ }^{4} D_{1 / 2}^{o}$ and $3 p^{4}\left[{ }^{3} P\right] 4 p^{4} D_{3 / 2}^{o}$ satellite states of $\mathrm{Ar}^{+}$in the photoionization of argon in the energy range of $36.120 \mathrm{eV}$ to $36.520 \mathrm{eV}$. The thresholds of formation of the ${ }^{4} D_{1 / 2}^{o}$ and ${ }^{4} D_{3 / 2}^{o}$ states are 35.403 and $35.370 \mathrm{eV}$, respectively, relative to the Ar ground state [4]. We have measured the relative cross sections for these states by measuring the characteristic electron emission at the magic angle $\left(54.7^{\circ}\right)$ relative to the linear polarization axis of the vacuumultraviolet (vuv) synchrotron radiation. The experiments were performed on the beamline 10.0.1.2 of the Advanced Light Source (ALS) at the Lawrence Berkeley National Laboratory (LBNL). Linearly polarized photons were made to collide with Ar in a gas cell. The electrons emitted at the magic angle were energy analyzed using a Scienta Gammadata SES-200 hemispherical electrostatic analyzer. The beamline was operated at high resolution for these experiments with a photon band pass of $5 \mathrm{meV}$. The electron energy analyzer utilized a pass energy of $2 \mathrm{eV}$. A typical measured-electron energy spectrum for electrons emitted at the magic angle and at the ionizing-photon energy of $36.246 \mathrm{eV}$ is shown in Fig. 1. As can be seen from Fig. 1, different fine-structure peaks were easily resolved in the photoelectron spectra. From known values of their thresholds of formation [4], the energy spacing of 66, 39, 82, 37, and $33 \mathrm{meV}$ between the peaks (from low to high electron energies) are used to identify the final fine structure of the residual ion. The analyzer system simultaneously acquired data for several electron energies so that beam intensity and target density variations did not affect the relative intensity of the peaks of the spectrum that are close in energy.

The two largest peaks in the spectrum of Fig. 1 are associated with the ${ }^{4} D_{1 / 2}^{o}$ and ${ }^{4} D_{3 / 2}^{o}$ states of interest. We note that the peak heights and areas of the quartet states are significantly greater than the doublets. For this particular ionizing photon energy our measurements show that the cross sections for the two quartet states, which are proportional to the area under each peak, are significantly larger than the integrated area of the dipole-allowed doublet states, ${ }^{2} D_{5 / 2}^{o},{ }^{2} D_{3 / 2}^{o}$, ${ }^{2} P_{3 / 2}^{o}$, and ${ }^{2} P_{1 / 2}^{o}$.

To quantitatively illustrate the difference between the quartets and doublets, the ratio of the areas of the peaks

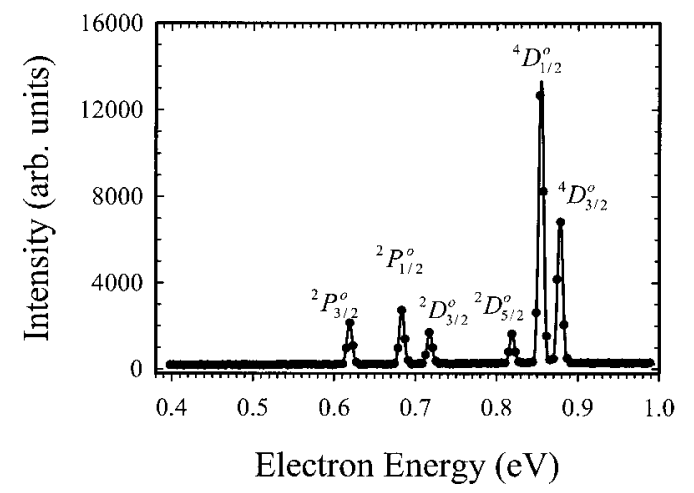

FIG. 1. Electron energy spectrum taken at the ionizing photon energy of $36.246 \mathrm{eV}$. Data points are indicated by filled circles; the line is the calculated fit assuming Gaussian profiles for each peak. 

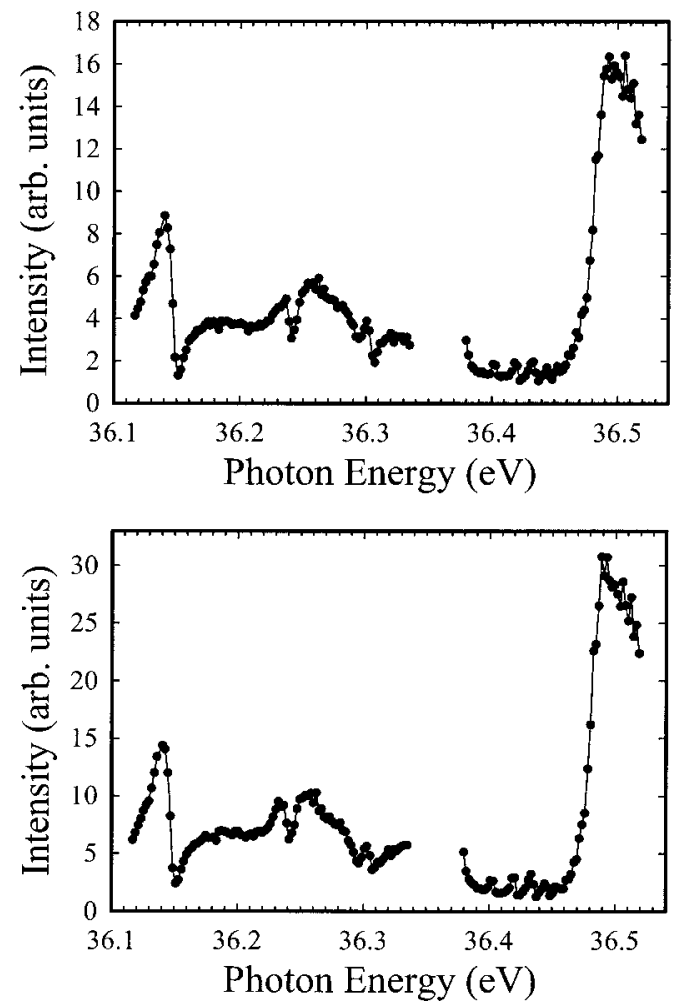

FIG. 2. Top graph (a) shows the integrated area of the peak in the photoelectron spectra corresponding to the ${ }^{4} D_{3 / 2}^{o}$ state while the bottom graph (b) shows the integrated area of the peak in the photoelectron spectra corresponding to the ${ }^{4} D_{1 / 2}^{o}$ state, both normalized to the same ${ }^{2} D_{5 / 2}^{o}$ state area.

associated with the ${ }^{4} D_{1 / 2}^{o}$ and ${ }^{4} D_{3 / 2}^{o}$ states to the ${ }^{2} D_{5 / 2}^{o}$ state have been plotted at different photon energies. The area of one electron peak can be expressed by the relationship

$$
A_{m}(1)=\varepsilon_{m}(1) \sigma_{0}(1)\left[1+\beta(1) P_{2}\left(\cos \theta_{m}\right)\right] / 4 \pi .
$$

The factor $\varepsilon_{m}$ encompasses the solid angle electron detector efficiencies at any given electron energy and at the magic angle. The quantities $\sigma_{0}(1), \sigma_{0}(2)$, etc., are the total cross sections for emitted electrons corresponding to peaks (1), (2), etc. At the magic angle of $\theta_{m}=54.7^{\circ}, P_{2}\left(\cos \theta_{m}\right)=0$. Therefore, the ratio of any two-peak areas at the magic angle is

$$
\frac{A_{m}(1)}{A_{m}(2)}=\frac{\varepsilon_{m}(1)}{\varepsilon_{m}(2)} \frac{\sigma_{0}(1)}{\sigma_{0}(2)} .
$$

The energy differences between the electrons associated with the ${ }^{4} D_{1 / 2}^{o}$ and ${ }^{4} D_{3 / 2}^{o}$ lines and those associated with the ${ }^{2} D_{5 / 2}^{o}$ state are $37 \mathrm{meV}$ and $70 \mathrm{meV}$. Since the energy of the photoelectrons is about $850 \mathrm{meV}$, we would expect a negligible difference in the efficiency of the electron energy analyzer for these two electron energies. Hence, the ratio of the peak intensities measured at the magic angle represents the ratio of the cross sections of the two quartet states to the doublet state. The intensities of the ${ }^{4} D_{1 / 2}^{o}$ and ${ }^{4} D_{3 / 2}^{o}$ peaks referenced to the ${ }^{2} D_{5 / 2}^{o}$ intensities are shown in Fig. 2 . We estimate the typical errors associated with the ratios to be about $9 \%$ for
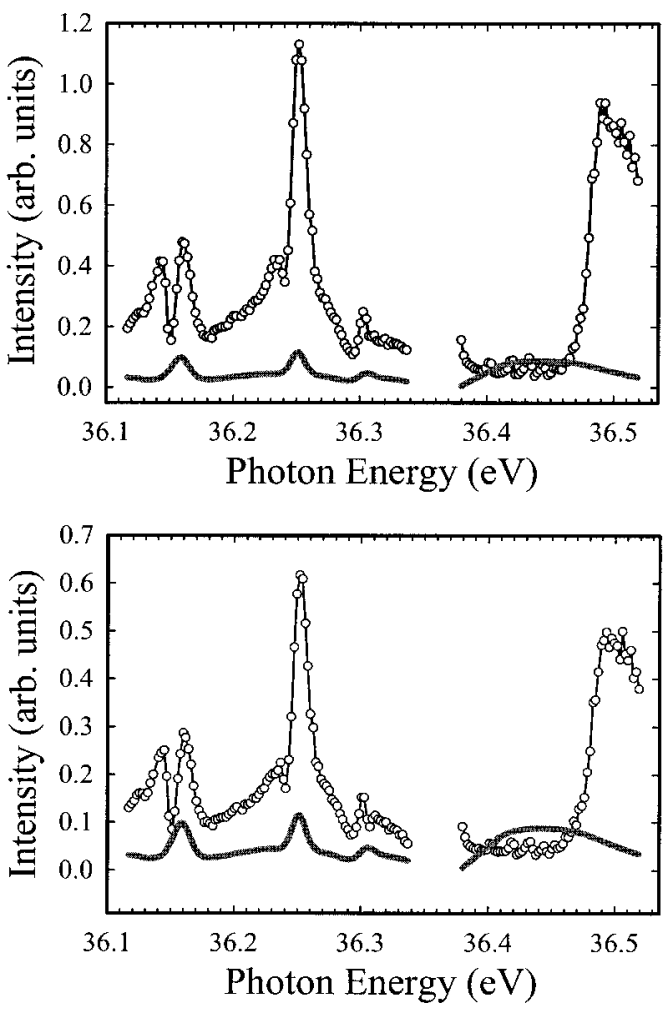

FIG. 3. The open circles show the data from Fig. 2 multiplied by the ${ }^{2} D_{5 / 2}^{o}$ fluorescence cross section over the same ionizing photon energy range. The top graph is for the ${ }^{4} D_{3 / 2}^{o}$ state and the bottom one is for the ${ }^{4} D_{1 / 2}^{o}$ state. The thicker line shows the ${ }^{2} D_{5 / 2}^{o}$ fluorescence cross section.

both Figs. 2(a) and 2(b). To take into account the energy variation of the reference intensity we multiplied the ratio shown in Fig. 2 by the ${ }^{2} D_{5 / 2}^{o}$ cross section, according to

$$
\sigma_{0}\left({ }^{4} D_{1 / 2}^{o}\right)=\frac{A_{m}\left({ }^{4} D_{1 / 2}^{o}\right)}{A_{m}\left({ }^{2} D_{5 / 2}^{o}\right)} \sigma_{o}\left({ }^{2} D_{5 / 2}^{o}\right)
$$

where $\sigma_{0}\left({ }^{2} D_{5 / 2}^{o}\right)$ is the fluorescence cross section determined in a separate experiment from measurements of the characteristic total intensity of $\operatorname{Ar}^{+}\left\{3 p^{4}\left[{ }^{3} P\right] 4 p^{2} D_{5 / 2}^{o}\right\}$ to $\operatorname{Ar}^{+}\left\{3 p^{4}\left[{ }^{3} P\right] 4 s^{2} P_{3 / 2}^{o}\right\}$ transition at $\lambda=488.0 \mathrm{~nm}$, using the apparatus previously described [5]. Since the fluorescence measurements have constant efficiency from threshold to any energy, no efficiency correction is needed for this multiplication. The contribution of the statistical errors associated with the fluorescence (about 2\%) to Figs. 3(a) and 3(b) is insignificant and a typical error bar for Figs. 3(a) or 3(b) is still about $9 \%$.

These results are shown in Fig. 3. Also shown is the relative-measured ${ }^{2} D_{5 / 2}^{o}$ cross section given in arbitrary units. Since the fluorescent radiation collection efficiency factors are independent of the ionizing energy, the relative quartet cross sections are now on the same scale as the ${ }^{2} D_{5 / 2}^{o}$ cross section. The gap in the data of Figs. 2 and 3, of approximately $50 \mathrm{meV}$ of width, is due to the allocation of beam time at ALS and would not change any discussion or conclusions of this paper. The structures observed on the fluores- 
cence data are due to the doubly excited states of Ar. Their identification was tabulated by Madden, Ederer, and Codling [6]. Their observations in fluorescence experiments were discussed in Ref. [5] over an energy range that includes the range of the present paper [5].

We have found two quartet states of $\left(\mathrm{Ar}^{+}\right)^{*}$ formed in photoionization where the decay of the intermediate state of Ar is dominated by transitions to the quartet states, ${ }^{4} D_{1 / 2}^{o}$ and ${ }^{4} D_{3 / 2}^{o}$. We have determined their cross sections relative to the dipole-allowed doublet states of $\mathrm{Ar}^{+}$over a $400-\mathrm{meV}$ energy range. We find that they are an order of magnitude larger than the doublet cross sections over most of this energy range. The cross sections for the ${ }^{4} D_{1 / 2}^{o}$ and ${ }^{4} D_{3 / 2}^{o}$ states ex-

[1] H. W. van der Hart and C. H. Greene, J. Phys. B 32, 4029 (1999).

[2] G. Mentzel, K. H. Schartner, O. Wilhelmi, B. Magel, U. Staude, F. Vollweiler, S. Lauer, H. Liebel, H. Schmoranzer, V. L. Sukhorukov, and B. M. Lagutin, J. Phys. B 31, 277 (1998).

[3] O. Yenen, K. W. McLaughlin, T. J. Gay, J. R. Machacek, K. Aflatooni, and D. H. Jaecks (unpublished). hibit resonancelike behavior similar to the doublets. The fact that these two quartet states are larger by an order of magnitude suggests that within the model of singlet-triplet spinorbit mixing of states in $\mathrm{Ar}^{* * *}$, the triplet component of the state is larger than the singlet component. This counterintuitive result has not been theoretically addressed. It is clear that the description of the photoprocess in Ar is filled with many interesting and subtle phenomena that deserve further study.

This work has been supported by the National Science Foundation through Grant No. NSF-9731869. We thank the entire staff of ALS for their dedicated work.
[4] L. Minnhagen, Ark. Fys. 25, 203 (1963).

[5] K. W. McLaughlin, O. Yenen, D. H. Jaecks, T. J. Gay, M. M. Sant'Anna, D. Calabrese, and B. Thaden-Jordan, Phys. Rev. Lett. 88, 123003 (2002).

[6] R. P. Madden, D. L. Ederer, and K. Codling, Phys. Rev. 177, 136 (1969). 